\title{
High Tissue Factor Microparticle Level in Major Thalassemic Patients with Pulmonary Hypertension
}

\author{
Dimmy Prasetya, Pandji Irani Fianza, Erwan Martanto, Teddy Arnold Sihite \\ Department of Internal Medicine Faculty of Medicine Universitas Padjadjaran \\ Dr. Hasan Sadikin General Hospital Bandung, Indonesia
}

\begin{abstract}
Objective: To analyze the correlation between tissue factor microparticles (TF-MP) levels and pulmonary hypertension (PH) in adult thalassemic patients.

Methods: This study was conducted from September to October 2018, using secondary and primary data. The secondary data consisted of the PH parameter, which was retrieved from a 2017 previous study entitled 'Clinical Characteristic and Complication due to Iron Overload in Thalassaemic Patients'in 2017 while the primary data were the TF-MP which were obtained from the analysis of frozen serum of the same population using ELISA method. The mean pulmonary arterial pressure (mPAP) values were obtained from echocardiography results and PH was defined as mPAP $>25$ $\mathrm{mmHg}$.
\end{abstract}

Results: Seven (16.7\%) major thalassemic patients experienced PH. The median values of TF-MP levels were higher among major thalassemic patients with PH when compared to the non-PH patients (1569 vs $11.5 \mathrm{pg} /$ $\mathrm{dL} ; \mathrm{p}=0.023$ ). No significant difference was observed in the median TF-MP levels between subjects with splenectomy and subjects without splenectomy (11.6 vs $12.3 \mathrm{pg} / \mathrm{dL} ; \mathrm{p}=0.44)$. There was also no difference in mPAP values between subjects with splenectomy and subjects without splenectomy (18.0 vs $17.0 \mathrm{mmHg}$; $=0.663$ ). When the median TF-MP levels among major thalassemic patients were analyzed in terms of correlation with transfussion level, no statistically significant difference was seen between subjects who received sufficient transfusions $(\geq 180 \mathrm{~mL} / \mathrm{kgbb} /$ year $)$ and those who received insufficient transfusions ( $<180 \mathrm{~mL} / \mathrm{kgbb} /$ year $)(r=0.138 ; \mathrm{p}=0.390)$.

Received: Conclusions: There is a positive correlation between the TF-MP levels and March 11, $2019 \quad \mathrm{PH}$ in adult major thalassemic subjects.

Revised:

September 17, 2019

Keywords: Major thalassemia, pulmonary hypertension, tissue factor microparticles

Accepted:

October 19, 2019 pISSN: 2302-1381; eISSN: 2338-4506; http://doi.org/10.15850/ijihs.v7n2.1658

IJIHS. 2019;7(2):88-95

\section{Introduction}

Homozygous $\beta$-globin carrier defects in thalassemia patients can give rise to several complications due to hemolysis, chronic anemia, and continuous transfusions. In

Correspondence:

Dimmy Prasetya,

Department of Internal Medicine Faculty of Medicine Universitas Padjadjaran/Dr. Hasan Sadikin General Hospital Bandung, Jalan Psteur No. 38 Bandung, West Java, Indonesia

e-mail: dimmy.prasetya@unpad.ac.id the last few decades, major thalassemia patients can live longer with the availability of blood transfusion and iron chelation. With the longer survival, the previously unrecognized complications associated with hypercoagulability become visible. ${ }^{1,2}$

Complications associated with hypercoagulability appear to be subclinical, marked only by changes in the hemostasis parameters, until thromboembolic processes such as stroke, transient ischemic attack, deep vein thrombosis (DVT), and pulmonary embolism occur. Pulmonary hypertension $(\mathrm{PH})$ in thalassemia is included in the 5 th class 
of the PH classification of the 2013 American College of Cardiology and is considered to happen via multifactorial mechanisms. It is considered as the complication that becomes the main factor of morbidity and mortality in these patients. Pulmonary hypertension involves several mechanisms such as endothelial dysfunction, inflammation, and chronic thromboembolism. The mechanism of thromboembolism in thalassemia patients with PH has not been clearly understood. ${ }^{3,4}$

The multifactorial mechanism requires microparticles to play distinctive roles in the cellular interactions related to $\mathrm{PH}$ pathogenesis. Microparticles are vesicles from the plasma membrane, ranging from 0.1 to 1 $\mu \mathrm{m}$ in size, that are released by cells through activation by their agonists, physical and chemical stresses, and apoptosis. Tissue factor microparticles (TF-MP) or circulating Tissue factor (cTF) is a protein released by cellular activation or apoptosis that plays an important role in producing thrombin in the propagation phase of the cell-based coagulation. Cellular activation in thalassemia is caused by negatively-charged cell membranes and hemolysis process disorders. Previous studies have reported that increased TF-MP levels are found in thalassemia patients, as well as in patients with 1st and 3rd group PH. ${ }^{5,6}$

No data available yet on the roles of TF-MP in thalassemia patients with PH. This study aimed to analyze the correlation between TF-MP level and PH in major thalassemia patients in Dr. Hasan Sadikin General Hospital, Bandung, West Java, Indonesia.

\section{Methods}

This was a cross-sectional quantitative study using numeric-numeric correlative statistical analysis conducted from September to October 2018. Subjects in the study were thalassemia patients treated at the Hematology-Medical Oncology Clinic, Internal Medicine Department, Faculty of Medicine, Universitas Padjadjaran/ Dr. Hasan Sadikin General Hospital Bandung. PH parameters were collected as secondary data from a previous study entitled ' Clinical Characteristic and Complication due to Iron Overload in Thalassaemic Patients ' which was conducted in 2017 while data on TF-MP were collected by testing the frozen serum of the same population of this 2017 study.

The inclusion criteria were thalassemia patients aged $\geq 14$ years who had received treatments and regular transfusions for more than two years after diagnosis, had echocardiographic data, and had their frozen serum stored. The exclusion criteria were intermediate thalassemia patients and patients with left ventricular or left atrial hypertrophy. Pulmonary hypertension $(\mathrm{PH})$ was measured using the GE Vivid S6 echocardiography device. Meanwhile, the severity of $\mathrm{PH}$ was determined by estimating the mean Pulmonary Arterial Pressure (mPAP) $(\geq 25 \mathrm{mmHg})$. The mPAP was measured while the patient was resting through the use of the Pulmonary Velocity Accelerated Time (PVaccT) calculation [mPAP $=80-($ PVaccT $/ 2)]$. Data on the MP-TF levels were collected through subject serum analysis using the ELISA method and the results were reported in $\mathrm{pg} / \mathrm{dL}$.

Before statistical analyses were performed, data normality testing was performed on numerical data, such as age, period of suffering from thalassemia, transfusion period, total transfusions volume, sufficiency of transfusions, ferritin levels, Hemoglobin (Hb) levels, thrombocyte counts, mPAP values, and TF-MP levels. The Shapiro-Wilk test was selected for this testing because the number of subjects was less than 50 . Data on age, ferritin levels, hematocrit percentages, thrombocyte counts, ferritin levels, period of suffering from thalassemia, sufficiency of transfusions, mPAP values, and MP-TF levels were not normally distributed $(p>0.05)$, while data on Hb level were normally distributed ( $p>0.05)$.

Normally distributed data were presented in mean and analyzed using Pearson correlation (r) test, while data that were not normally distributed were presented in median and analyzed using non-parametric Spearman correlation test (rs). Point biserial correlation (rpb) analysis was performed to observe the correlation between numerical and categorical data. Results were declared as significant if $\mathrm{p}<0.05$.

\section{Results}

Subject characteristics, including age, sex, ferritin levels, $\mathrm{Hb}$ levels, Ht percentages, thrombocyte counts, sufficiency of transfusions, numbers of subjects with $\mathrm{PH}$ or without PH, mPAP values, and TF-MP levels, are presented in Table 1 . The median age was 20 years old. More females were included in the study than males $338.1 \%$ versus $61.9 \%$ ). The median period of suffering from thalassemia was 198 months. The sufficiency of transfusion was $65.6 \mathrm{cc} / \mathrm{kgBW} /$ year while the mean $\mathrm{Hb}$ level, median thrombocyte count, and median ferritin level were $7.0 \pm 1.3 \mathrm{~g} / \mathrm{dL}$, 


\section{Table Study Subject Basic Characteristics}

\begin{tabular}{lc}
\hline \multicolumn{1}{c}{ Variable } & $\mathbf{n = 4 2}$ \\
\hline Age (years old), median (min-max) & $20(16-36)$ \\
Sex, n (\%) & \\
$\quad$ Male & $16(38.1)$ \\
$\quad$ Female & $26(61.9)$ \\
Period of suffering from thalassemia (months), median (min-max) & $198(68-291)$ \\
Group, n (\%) & \\
$\quad$ Splenectomy & $12(28.6)$ \\
$\quad$ Non-splenectomy & $30(71.4)$ \\
Hb (g/dL), mean \pm SD & $7.0 \pm 1.3$ \\
Hb category & \\
$\quad<9$ g/dL & $40(95.2)$ \\
$\quad \geq 9$ g/dL & $2(4.8)$ \\
Ht (\%), median (min-max) & $22.0(9.1-51.9)$ \\
Thrombocyte count (x1000/mm $\left.{ }^{3}\right)$, median (min-max) & $137(18-903)$ \\
Ferritin ng/dL, median (min-max) & $3940(948-14030)$ \\
Ferritin category ng/dL, n (\%) (ng/dL) & \\
$\quad<2,500$ & $13(31.0)$ \\
$\quad \geq 2,500$ & $29(69.0)$ \\
Sufficiency of transfusion (mL/kgbb/year), median (min-max) & $65.6(0.0-339.9)$ \\
Sufficiency of transfusion category, n (\%) & $\mathrm{n}=41$ \\
$\quad \geq 180$ mL/kgbb/year & $5(11.9)$ \\
$\quad<180$ mL/kgbb/year & $36(85.7)$ \\
PH and non-PH group & $7(16.7)$ \\
$\quad$ PH & $35(83.3)$ \\
$\quad$ Non-PH & $17.0(9.0-43.0)$ \\
MPAP (mmHg), median (min-max) & $12.1(5.3-2615.2)$ \\
\hline
\end{tabular}

Note: $\mathrm{SD}=$ Standard deviation, $\mathrm{n}=$ total, $\%=$ Percentage

137,000 , and 3,940 ng/dL, respectively.

The subjects characteristics including the age, sex, ferritin levels, Hb levels, Ht percentages, thrombocyte counts, sufficiency of transfusions, numbers of subjects with $\mathrm{PH}$ or without $\mathrm{PH}, \mathrm{mPAP}$ values, and TF-MP levels can be seen in Table 1 below. The median of age was 20 , consisting of males $(38.1 \%)$ and females $(61.9 \%)$. The median of period of suffering from thalassemia was 198 months. The sufficiency of transfusions was $65,6 \mathrm{cc} /$ $\mathrm{kgbb} /$ year, the mean of $\mathrm{Hb}$ level was $7.0 \pm 1.3$ $\mathrm{g} / \mathrm{dL}$, the median of thrombocyte count was
137.000 , and the median of ferittin level was $3940 \mathrm{ng} / \mathrm{dL}$.

When the correlation between MP-TF level and mPAP value was assessed using the Spearman correlation test with a 95\% confidence interval, it was revealed that the MP-TF level had a statistically significant correlation with the mPAP value $(\mathrm{r}=0.481$; $\mathrm{p}=0.001)$. $(\mathrm{r}=0.481 ; \mathrm{p}=0.001)$.

\section{Discussion}

This study presented a correlation between 


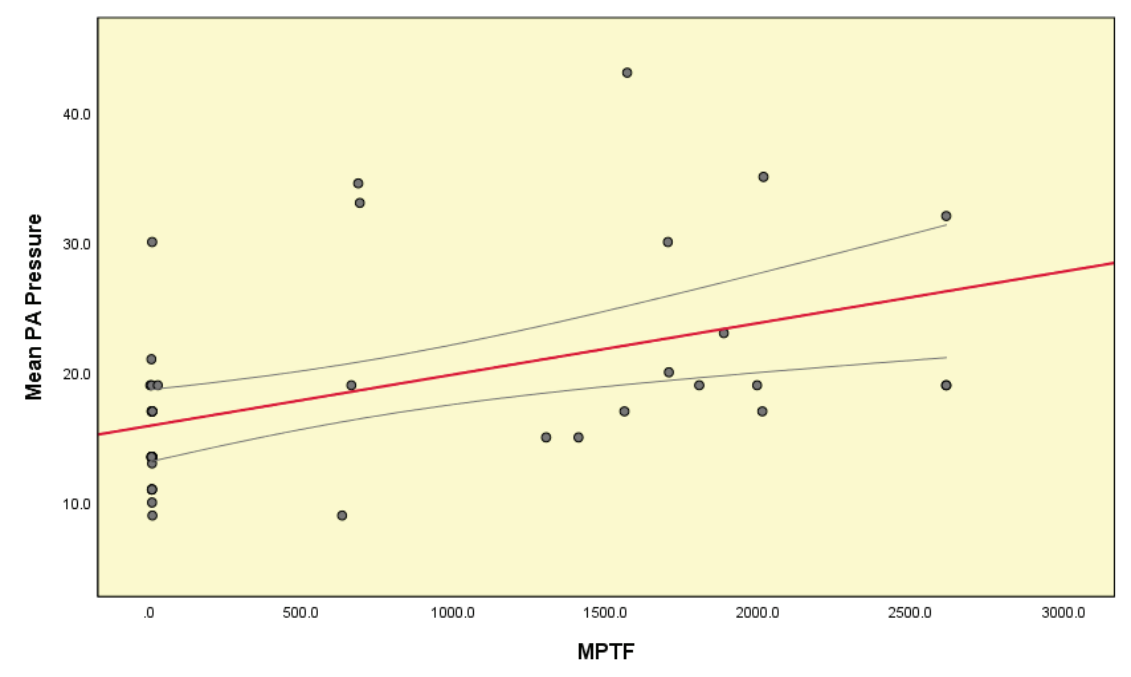

Fig 1 Scatter Diagram of Correlation between MP-TF Level and mPAP Value

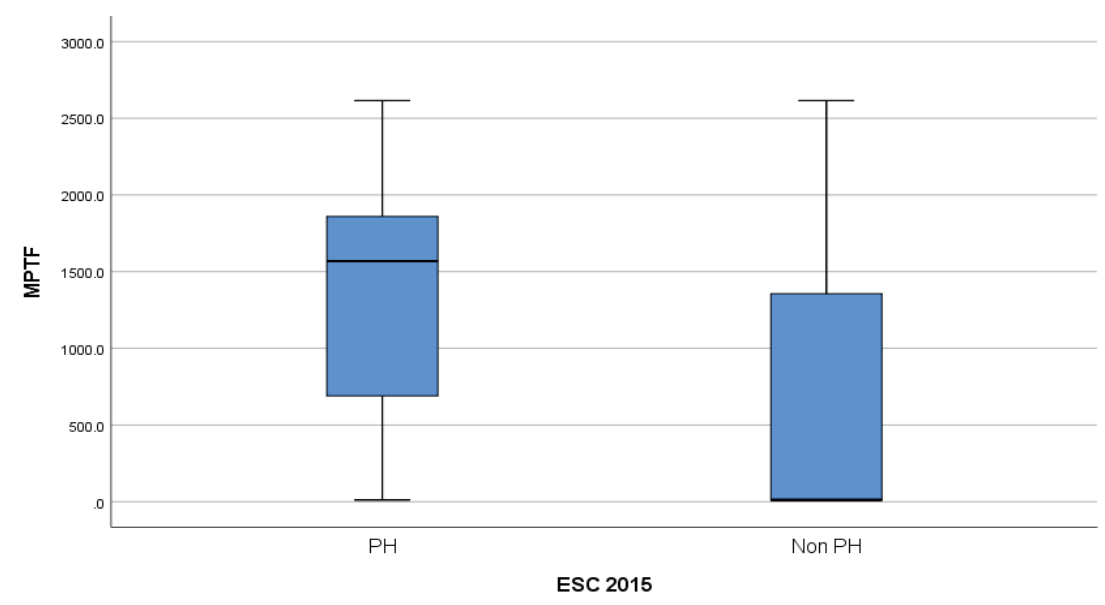

Fig. 2 Boxplot of Median MP-TF Level in PH and Non-PH Groups

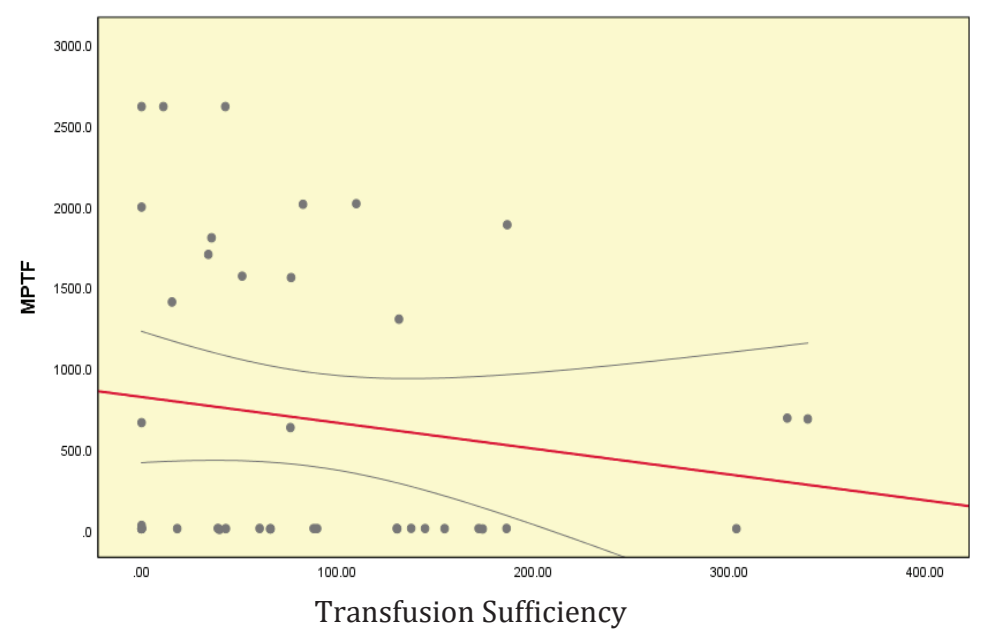

Fig. 3 Scatter Diagram of Correlation between Transfusion Sufficiency and TF-MP Level 


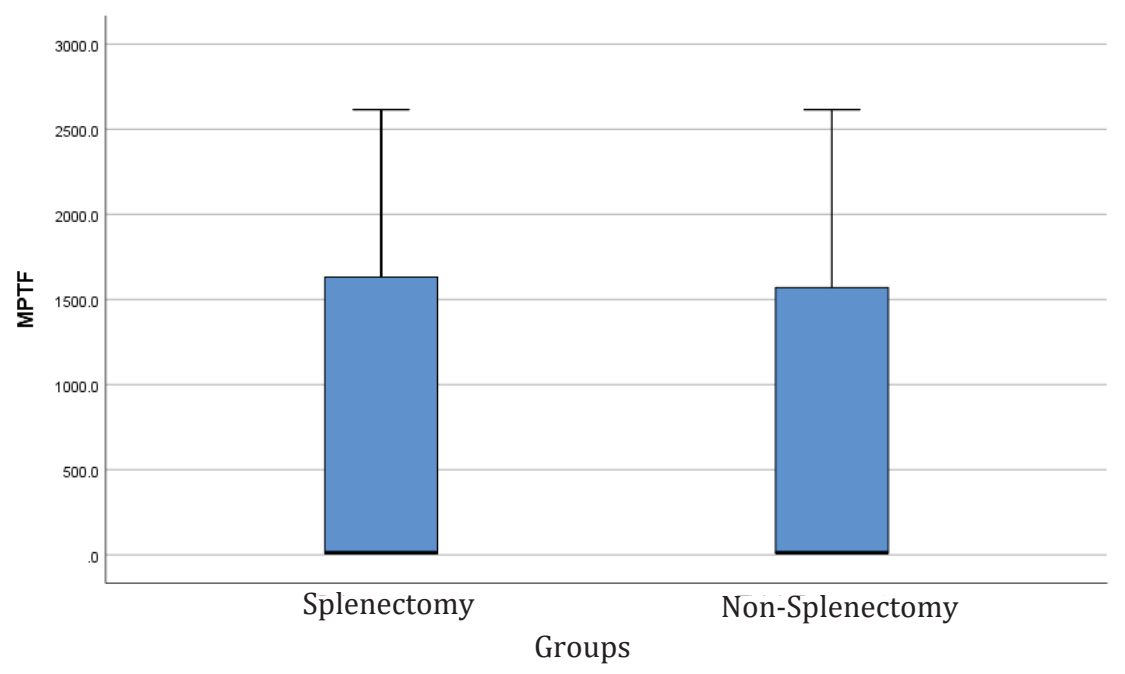

Fig. 4 Boxplot of TF-MP Level in Splenectomy and Non-splenectomy Groups

increased MP-TF levels and high mPAP values (moderate correlation; $\mathrm{r}=0.481 ; \mathrm{p}<0.001$ ). Based on the point biserial analysis to observe a direct correlation between the MP-TF level and the presence of $\mathrm{PH}$, a significant difference was observed between $\mathrm{PH}$ and non-PH subjects $(\mathrm{p}<0.05 ; \mathrm{rpb}=0.311)$.

A study conducted by Bakoubula et al. in 2008 that examined the correlation between MP-TF levels and PH by enrolling 20 subjects with 1st and 3rd group of $\mathrm{PH}$ discovered a statistically significant correlation between the MP-TF level and PH ( $p<0.001)$. Dhiel et al. in 2010 who studied 19 subjects with PH caused by chronic thromboembolism, idiopathic PH, rheumatic-related $\mathrm{PH}$, and chronic pulmonary disease (CPD)-related PH also found a positive correlation between procoagulant MP level and occurrence of PH in study subjects when compared to the normal control. ${ }^{7,8}$

Hypercoagulability complications in thalassemia patients are characterized by

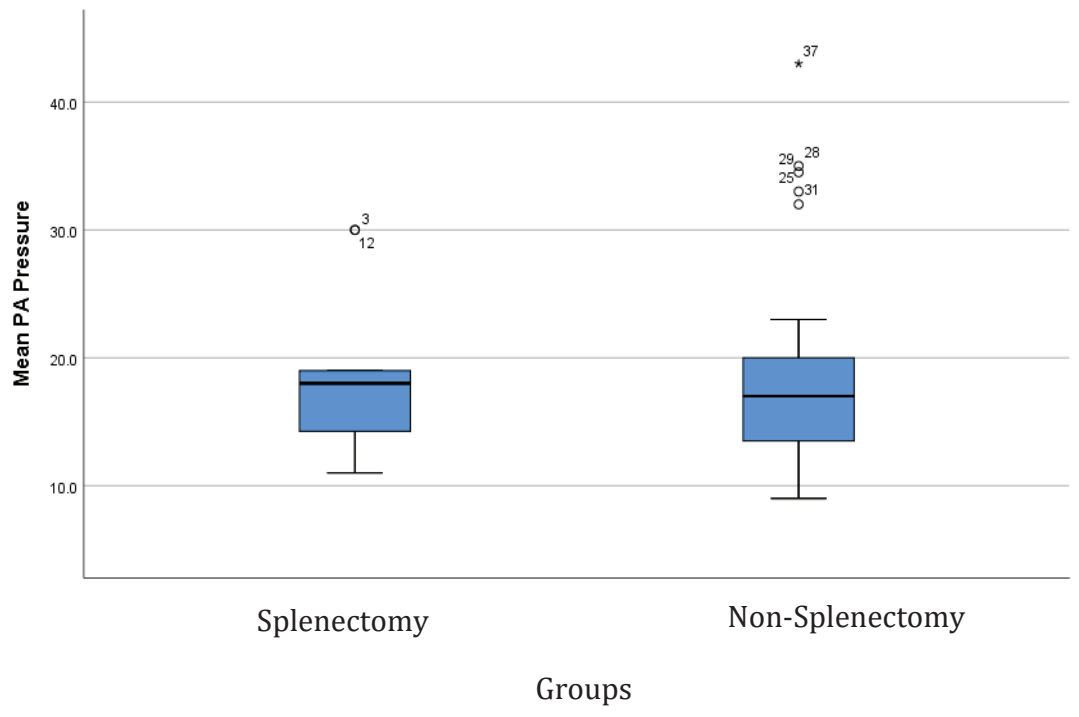

Fig. 5 Boxplot of mPAP Levels in Splenectomy and Non-splenectomy Groups 
subclinical defects marked by hemostasis parameter changes until the occurrence of thromboembolic events such as stroke, transient ischemic attack, DVT, and chronic pulmonary embolism. The PH in thalassemia can be included in the 5th group of the 2013 ACC classification. Pulmonary hypertension in thalassemia is caused by various factors and mechanisms, including pulmonary arteriole in situ thrombosis. Interactions among factors that play the roles in the occurrence of $\mathrm{PH}$ in major thalassemia patients require a medium such as procoagulant MP..$^{5,9}$

Thisstudyreveals a correlation between MPTF level and mPAP value in major thalassemia population. This study supports findings of previous studies and adds supporting data of in situ thrombosis process in the pathogenesis of the 5th group $\mathrm{PH}$ through the roles of procoagulant microparticles, such as TF-MP. ${ }^{5}$

The correlation between the TF-MP level and $\mathrm{PH}$ discovered in this study is considered moderate $(\mathrm{r}=0.481)$. This may relate to other mechanism pathways, such as the endothelial dysfunction pathway, triggered by decreased nitric oxide and inflammation. Endothelial dysfunction causes an imbalance in mediator production, which leads to vasoconstriction, smooth muscle hyperplasia, and pulmonary arterial remodeling. Other factors that can influence the occurrence of $\mathrm{PH}$ are not included in this study despite the possibility that they may also influence the significance of the correlation. Hence, this is considered a limitation in this study. ${ }^{5,10}$

Of all thalassemia patients in this study, $16.7 \%$ had PH. This is different from a previous study by Fayed et al. in 2017 which reported that $36 \%$ of major thalassemia children have $\mathrm{PH}$. The presence of $\mathrm{PH}$ in their study was determined through echocardiographic examination by calculating mPAP using a different formula. ${ }^{11}$

Based on the 2015 ESC, mPAP is not used for echocardiographic diagnosis of PH. Instead, the 2015 ESC uses the tricuspid regurgitant velocity (TRV) and other imaging techniques for assessing complications of $\mathrm{PH}$ in the right side of the heart. This method is more specific and widely used. The results are categorized as low, moderate, or high probability for $\mathrm{PH}$. This study uses secondary data from a previous study. However, the reference study did not use TRV calculation in the determination of $\mathrm{PH}$, which creates another limitation of this study. ${ }^{12}$

The median TP-MF level of the subjects of this study was $12.1 \mathrm{pg} / \mathrm{dL}$, which is much higher than the TP-MF level in normal people, which is less than $0.21 \mathrm{pg} / \mathrm{dL}$. The increase in the TF-MP level in major thalassemia patients in comparison to the normal population is also demonstrated in some previous studies. A higher level of TF-MP can also be found in several other diseases such as HIV, diabetes mellitus, and sickle cell disease. ${ }^{13,14}$

Thedeterminantfactorsofdefect in thalassemia are chronic hemolysis and ineffective erythropoiesis with iron overload which leads to complications in thalassemia, including hypercoagulability. Hemichrome precipitation due to thalassemic erythrocyte globin chain defect can cause heme disintegration, enhance intracellular labile iron, and produce reactive oxygen species, which lead to endothelial activation and dysfunction. ${ }^{10}$

The majority of the subjects in this study (95.2\%) had a pre-transfusion $\mathrm{Hb}$ level of $<9 \mathrm{~g} / \mathrm{dL}$, which was caused by insufficient transfusions. The median of transfusion sufficiency in this study was $65.6 \mathrm{cc} / \mathrm{kg} \mathrm{BW} /$ year, which is below the standard of $\geq 180$ $\mathrm{cc} / \mathrm{kg}$ BW/year. No significant correlation was observed between the insufficiency of transfusions and either TF-MP level and the presence of PH. A previous study by Hastuti in Dr. Hasan Sadikin General Hospital, Bandung, Indonesia in 2007 on major thalassemia patients discovered a correlation between the sufficiency of transfusions and the hypercoagulability index when examined by TEG. The insufficiency of transfusions can cause a higher level of thalassemic erythrocyte cells. ${ }^{15}$

The results of this study were different from the results reported by Agouti et al. in 2015 on 37 major thalassemia patients. Their study described higher TF-MP in subjects who received insufficient transfusions, which was assessed using the flow cytometry test. Flow cytometry test is considered to be more sensitive and has the ability to identify the cell origin of TF-MP. Literature stated that complications due to chronic hemolysis and ineffective hematopoiesis in relation to the thromboembolic processes are more frequently seen in intermediate thalassemia than in major thalassemia. Agouti's study does not include intermediate thalassemia patients so complications caused by insufficiency of transfusions do not influence the higher TFMP level and PH. ${ }^{16}$

Splenectomy in thalassemia patients triggers an increase in thrombocyte count, activation, and aggregation. These conditions may lead to a higher level of TF-MP derived 
from thrombocytes. Splenectomy is also associated with an increase in mPAP values in thalassemia patients and previous studies have discovered a higher $\mathrm{PH}$ incidence in patients with splenectomy when compared to those without splenectomy. ${ }^{11}$

The result of this study showed no significant correlation between splenectomy and increased TF-MP level and MPAP value. A previous study stated that the incidence of $\mathrm{PH}$ is higher in thalassemia patients who had undergone splenectomy. The difference in result is probably caused by the number of the subjects in the study which is lower (36 subjects) than in this study, along with the higher frequency (47.2\%) of PH in their study. The mean thrombocyte count in their study is $548.200 \pm 242.500 / \mathrm{mm}^{3}$, which is higher than in this study $\left(137.000 / \mathrm{mm}^{3}\right) .{ }^{11}$

Several limitations are noted in this current study, including PH examination using PvaccT calculation, which is less specific than other methods such as the TRV from echocardiography. This study does not analyze the origin of cells that activate and release TFMP, thus unable to describe the influence of sufficiency of transfusions on the TF-MP level. This study also does not analyze major factors that can influence the pathogenesis of $\mathrm{PH}$ from the endothelial dysfunction and inflammation pathways; hence, major determinants in the significance of the correlation between TFMP level and PH in major thalassemia patients cannot be discovered. ${ }^{17,18}$

In summary, this study proves that the TF-MP level influences in situ thrombosis in the mechanism of PH in major thalassemia patients. Further studies with cohort design and multiple regression analysis of other determinant factors in the endothelial dysfunction and inflammation pathways that might contribute to the mechanism of $\mathrm{PH}$ should be conducted. In major thalassemic patients with sufficient transfusions, it is not crucial to discover the origin of cells that release TF-MP using flow cytometry test.

\section{References}

1. Nienhuis AW, Nathan DG. Pathophysiology and clinical manifestations of the $\beta$-thalassemias. Cold Spring Harb Perspect Med. 2012;2(12):a011726.

2. Sabath DE. Molecular diagnosis of thalassemias and hemoglobinopathies: an ACLPS critical review. Am J Clin Pathol. 2017;148(1):6-15.

3. Hoeper MM, Bogaard HJ, Condliffe R, Frantz
R, Khanna D, Kurzyna M, et al. Definitions and diagnosis of pulmonary hypertension. J Am Coll Cardiol. 2013;62(25 Suppl):D42-50.

4. Fraidenburg DR, Machado RF. Pulmonary hypertension associated with thalassemia syndromes. Ann N Y Acad Sci. 2016;1368(1): 127-39.

5. Amabile N, Guignabert C, Montani D, Yeghiazarians Y, Boulanger CM, Humbert M. Cellular microparticles in the pathogenesis of pulmonary hypertension. Eur Res J. 2013;42(1):272-9.

6. Barteneva NS, Fasler-Kan E, Bernimoulin M, Stern JN, Ponomarev ED, Duckett L, et al. Circulating microparticles: square the circle. BMC Cell Biol. 2013;14(1):23.

7. Bakouboula B, Morel O, Faure A, Zobairi F, Jesel L, Trinh A, et al. Procoagulant membrane microparticles correlate with the severity of pulmonary arterial hypertension. Am J Respir Crit Care Med. 2008;177(5):536-43.

8. Diehl P, Aleker M, Helbing T, Sossong V, Germann $M$, Sorichter $S$, et al. Increased platelet, leukocyte and endothelial microparticles predict enhanced coagulation and vascular inflammation in pulmonary hypertension. J Thromb Thrombolysis. 2011;31(2):173-9.

9. Abosdera MM, Almasry AE, Abdel-Moneim ES. Coagulation defects in thalassemic patients. Pediatr Neonatol. 2017;58(5):421-4.

10. Elsayh KI, Zahran AM, El-Abaseri TB, Mohamed AO, El-Metwally TH. Hypoxia biomarkers, oxidative stress, and circulating microparticles in pediatric patients with thalassemia in upper Egypt. Clin Appl Thromb Hemost. 2014;20(5):536-45.

11. Fayed MA, Abdel-Hady HE-S, Hafez MM, Salama OS, Al-Tonbary YA. Study of platelet activation, hypercoagulable state, and the association with pulmonary hypertension in children with $\beta$-thalassemia. Hematol Oncol Stem Cell Ther. 2018;11(2):65-74.

12. Galiè $N$, Humbert $M$, Vachiery J-L, Gibbs S, Lang I, Torbicki A, et al. 2015 ESC/ERS guidelines for the diagnosis and treatment of pulmonary hypertension: the joint task force for the diagnosis and treatment of pulmonary hypertension of the European Society of Cardiology (ESC) and the European Respiratory Society (ERS): endorsed by: Association for European Paediatric and Congenital Cardiology (AEPC), International Society for Heart and Lung Transplantation (ISHLT). Eur Heart J. 
2016;37(1):67-119.

13. Baker JV, Hullsiek KH, Bradford RL, Prosser R, Tracy RP, Key NS. Circulating levels of tissue factor microparticle procoagulant activity are reduced with antiretroviral therapy and are associated with persistent inflammation and coagulation activation among HIV positive patients. J Acquir Immune Defic Syndr. 2013;63(3):367-71.

14. Singh A, Boden G, Homko C, Gunawardana J, Rao AK. Whole-Blood tissue factor procoagulant activity is elevated in type 1 diabetes and effects of hyperglycemia and hyperinsulinemia. Diabetes Care. 2012;35(6):1322-7.

15. Hastuti TS. Hubungan volume darah transfusi dengan koagulabilitas pada penderita thalassemia yang mendapat transfusi berkala dan belum menjalani splenektomi [Thesis]. Bandung: Fakultas Kedokteran Universitas Padjadjaran, 2011.

16. Agouti I, Cointe S, Robert S, Judicone C, Loundou A, Driss F, et al. Platelet and not erythrocyte microparticles are procoagulant in transfused thalassaemia major patients. $\mathrm{Br} \mathrm{J}$ Haematol. 2015;171(4):615-24.

17. Parasuraman S, Walker S, Loudon BL, Gollop ND, Wilson AM, Lowery C, et al. Assessment of pulmonary artery pressure by echocardiography-a comprehensive review. . Int J Cardiol Heart Vasc. 2016;12:45-51.

18. Anthi A, Orfanos SE, Armaganidis A. Pulmonary hypertension in $\beta$ thalassaemia. Lancet Res Med. 2013;1(6):488-96. 